\section{TORRES E ICEBERGS}

\author{
Emilio F. Martínez de Velasco* \\ Dr. Arquitecto
}

504.8

\section{sinopsis}

Se ofrece en este articulo una visión personal del proyectista de la torre de TVE, en Madrid, pasando revista a todos los aspectos que han incidido en el resultado estetico finals y el papel del arquitecto dentro del equipo pluridisciplinar de técnicos de diferentes especialidades, que aparece en palabras del autor como la punta de un iceberg de grandes dimensiones, pero no por esto su intervención es menos importante, ya que si bien el diseño es en este caso un problema sobredeterminado, por los condicionantes del entormo y sobre todo tecnológicos, es preciso superar esta barrera para conseguir que los elementos que componen el conjunto, huste, plataformas, cubiertas y mástill formen una unidad plástica satisfactoria.

... "La mayor parte de los icebergs tiene una densidad especifica de 0,9 y por ello, seis séptimas partes de su masa quedan bajo la superficie del mar"...

$$
\text { E. Britannica (Vol. 9) }
$$

Creo que la sistemática dificultad que experimento, siempre que he de hablar o escribir en relación con la Torre de Radiotelevisión, no es explicable -0 , al menos, no solamente-. en función de la evidente ausencia de las habituales ( $y$, a veces, confortables) coartadas proyectuales de un encargo, digamos, normal. Pienso, más bien, que podria entenderse como consecuencia de una especie de "sindrome de lceberg", que si no supone necesariamente una ruptura absoluta entre arquitecto y obra, si traduce, mal que bien, una relación muy compleja entre ambos, una relación constituida, en porcentajes constantemente variables, por un razonable afecto y una menos razonable irritación o rechazo. Me explicaré.

En primer lugar, nuestra Torre no es cuantitativamente importante, dentro del conjunto de infraestructuras realizadas por RTV con motivo del Mundial-82. De hecho y referida solamente a los trabajos a cargo de la Subdirección de Arquitectura, constituye - con curiosa exactitud-esa séptima parte visible de lo que podriamos denominar, para entendernos, el "iceberg de inversiones" en edificación.

* Jefe de la Subdirección de Arquitectura de RTVE.

Autor del proyecto básico y co-director de desarrollo de provecto ejecución de obra de la Torre RTVE - Madrid.
Por otra parte, en relación con un imaginario "iceberg de esfuerzos", el incuestionable impacto popular (y quizá profesional) de la Torre, asume unas cuotas de representación tan previsibles como desproporcionadas respecto de esas ocultas seis séptimas partes de trabajo, de angustias, de decisiones, que ha comportado la realización simultánea de varios proyectos y obras de gran volumen, en las mismas condiciones de riesgo (1) y urgencia.

Por último y quizá éste sea el aspecto más significativo del sindrome en cuestion, me parece a mi que la ligura del arquitecto en una actuación de este especialisimo carácter, no es -en el mejor de los casos - otra cosa que la punta de "iceberg de profesionales", del equipo pluridisciplinar que, para bien o para mal, la han hecho posible. Felizmente, aqui, en la parte visible que mantiene la desproporción con la "sumergida", no está solo el arquitecto. Precisamente, otros padres de la criatura exponen en las páginas de esta misma revista todos - o casi- los datos técnicos manejados en el desarro110 de proyecto y en la construcción del artefacto.

En definitiva, y para quien esto suscribe, la reflexión sobre sus proplas y complicadas relaciones con la Torre representa siempre un cierto esfuerzo de aceptación. Es algo asi como la actilud ante un hijo demasiado brillan te, mucho más que el resto de la prole (y de cuya paternidad no se está muy seguro).

En función de ese efecto/rechazo, de ese "sindrome de iceberg" al que he aludido y aprovechando que otros más capacitados han cargado con el trabajo duro de explicar los pormenores técnicos del tema (que siempre son más aburridos de narrar, pero mucho más interesantes de leer), contaré a quienes tengan la santa paciencia de llegar al final, unos cuantos aspectos relacionados con las parcelas de proyecto y obra en que mi responsabilidad ha sido más directa y hasta quizá me atreva a reflexionar sobre los resultados de la experien. cia. Todo ello, de forma ciertamente desordenada y - para tranquilidad del lector- breve.

Obviamente, la primera -en el tiempo- responsabili. dad fue el diseno de la Torre, pero éste es un tema que no se debe mitificar (2). En efecto, se trata siempre de un problema sobredeterminado, ya que al prácticamente obligado manejo del circulo para las secciones horizontales hay que anadir un sinfin de condicionantes (previamente establecidos por los planificadores) para las cotas de plataformas, los diámetros minimos de las plantas, las alturas libres, etc., etc. En este caso, el Arquitecto pudo "colar" un nivel más de los inicialmente previstos (3), definir un perfil para la "cesta", inventar una pasarela perimetral ablerta y proponer un sistema estructural para las plantas cerradas y su enlace con ol fuste, que - sorprendentemente- resulto tan viable como para no requerir modificaciones sustanciales durante el proceso de cálculo y dimensionamiento (4). 


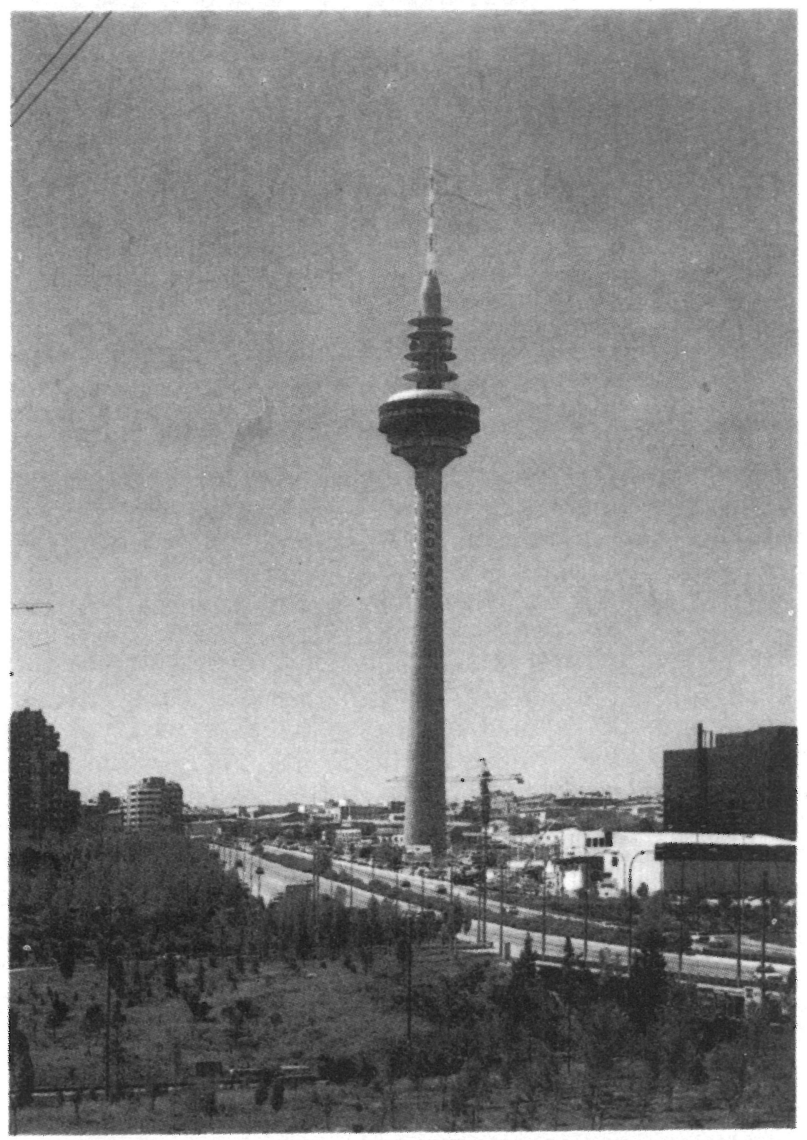

En resumen, después de muy pocos esquemas previos (pero mediando la croquización de infinidad de vistas "desde abajo", es decir desde la óptica de un ser humano), el trazado definitivo, idéntico al después construido, se convirtió, junto con un mínimo de especificaciones técnicas, en el Proyecto Básico, del que asumo la plena responsabilidad y al que, en Otoño de 1980, le fue concedida la licencia de construcción.

En cuanto a los materiales propuestos, no hay mucho que decir. Además del omnipresente hormigón (que, después de alguna vacilación, dejamos tal y como iba saliendo del molde), cabría mencionar dos decisiones no totalmente objetivas: acero "corten" para el cerramiento de la cesta y poliéster (reforzado con fibra de vidrio) para la cubierta de la última planta cerrada. En el primer caso, se trató de una opción muy personal que, desde el punto de vista práctico justifiqué en términos de mantenimiento (5), pero que, en el fondo, reflejaba mi aspiración de que aquello "envejeciera con la ciudad" (6). En el segundo, me pareció el modo más razonable de obtener paneles curvos - tóricos, para ser precisos - de un peso limitado (que, curiosamente y desde luego a posteriori, han resultado idóneos por su permeabilidad a las señales de video).

El citado proyecto, junto con un a veces genérico y a veces específico Pliego de Bases Técnicas, sirvió de base para el concurso de "desarrollo de proyecto y ejecución de obra" que se adjudicó con mucho retraso. Este retraso obligó a simultanear, aún más de lo previsto, ambos procesos, dando origen a alguna situación divertida, tal como la llegada "in extremis" de las últimas comprobaciones del cálculo, momentos antes de iniciarse el hormigonado de la losa de cimentación (7).
La construcción del fuste y plataformas se desarrolló sin particulares contratiempos, de forma ajustada al apretado planning impuesto, algo que hay que anotar en el haber de los adjudicatarios. En esta fase, las empresas en liza, sus departamentos de cálculo y sus especialistas en elevación de estructuras de este tipo tuvieron un protagonismo superior al de la dirección facultativa y es obligado señalar que a la falta de precedentes estrictos en nuestro pais (una chimenea se parece a una torre, pero no es una torre) se sumaron las dificultades adicionales derivadas de las limitaciones económicas, limitaciones que llevaron en muchos casos a suplir deseables medios auxiliares mediante "inventos" menos probados pero incuestionablemente ingenio$\operatorname{sos}(8)$.

De esta etapa, retengo $\mathrm{mi}$ inacabable pasmo ante el comportamiento del encofrado deslizante, la espectacular preparación (en el suelo) y posterior elevación y colocación del encofrado de la "copa" y sobre todo, la fascinación ante el vertido nocturno de los casi cinco millones de kilos de hormigón de la losa de cimentación (9).

La estructura de las plataformas abiertas y cerradas, brillante pero más convencional, el diseño de las instalaciones, que controlamos muy directamente, la esperada aparición de la "piel" de la cesta, a la que decidimos incorporar unos canalones de los que se espera eviten que el óxido del "corten" termine camuflando el fuste, la ampliamente debatida fijación de los prefabricados de poliéster y la más bien industrial - para entendernos- ejecución de los acabados interiores, completaron la penúltima fase de los trabajos. Un buen día, la ya familiar jaulilla que nos transportaba, rápida y emocionantemente, hasta lo alto del fuste, fue reemplazada por un montacargas que, si bien no nos obligaba a viajar dolorosamente encorvados, empleaba en el recorrido un tiempo a todas luces excesivo (10). Un poco antes, el mástil metálico para soporte de sistemas radiantes coronaba el invento. Gracias a la ampliación de su longitud en 15 metros sobre los 25 inicialmente previstos, habiamos superado la barrera simbolica de los 200 metros (11).

En febrero de 1982, sólo un año después de que las primeras máquinas iniciaran el vaciado para la cimentación, alguien decidió que el hasta entonces "piruli" se llamaria Torrespaña y casi, al mismo tiempo, los técnicos de RTVE se abalanzaron sobre el nuevo juguete y comenzaron a llenarlo de sofisticada cacharreria electró nica. Los que alli habiamos trabajado hasta entonces, abrumados, comprendimos que ambos acontecimientos, no del todo inconexos, señalaban el fin de nuestro cometido ( $y$ comenzaban otro iceberg...)
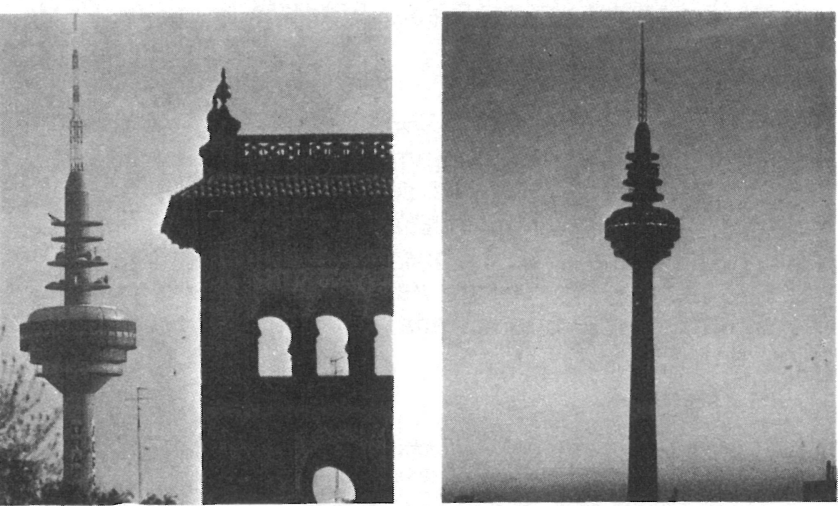
Al principio de estas notas he prometido -o amenazado, según se mire - algunas reflexiones sobre el proceso y los resultados. Ahi van.

En cuanto al diseño, ni me siento particularmente feliz, ni tampoco preocupado. Creo que no hay torres ubonitas", sino, simplemente torres. En cambio, si me parece que hay torres "feas", concretamente aquellas que, de algún modo, disfrazan su condición (pienso que éste no es el caso). Por 10 que respecta al impacto urbano, la ubicación de la Torre RTVE no afecta especialmente al perfil de la ciudad y de cualquier modo, para el madrileño que pase por las inmediaciones (no es fácil, no hay aceras), el altísimo recién llegado no será más agresivo que cualquier aglomeración especulativa de viviendas u oficinas o que la propia M-30, para la que supone una respuesta no desproporcionada.

Durante el proceso, todos hemos aprendido. Los que ya sabian, han contrastado sus conocimientos - necesariamente teóricos en este campo concreto- y los demás hemos vivido ( $y$ con suerte, aprovechado) una experiencia infrecuente... Se podrán mejorar soluciones y sistemas, sustituir riesgos por opciones ahora comprobadas, dilatar plazos de ejecución y mejorar acabados y diseños localizados (12), pero en conjunto y hasta donde yo sé, ninguno de los que hemos colaborado ha resultado traumatizado, a pesar de las prisas, las limitaciones económicas o la responsabilidad agobiante a veces.

Las instalaciones, como los acabados, se han resentido, en cierto modo, de la falta de tiempo para un diseño aún más especifico. Son, sin embargo, adecuadas y en contra de 10 que pueda pensarse, no particularmente sofisticadas, al menos en relación con los standards que habitualmente manejamos en radio y TV. Del ascensor/montacargas no puede, en cambio, decirse $10 \mathrm{mis}$ - mo y si algún lector tiene in mente el proyecto y/o construcción de torres de este estilo, le recomendaria que trabajase sobre velocidades un punto -aunque sólo sea uno- superiores a las que se derivan de las escuetas necesidades de tráfico: más de dos minutos de trayecto minimo son muchos segundos de tedio.

Por último, hay que decir que la Torre ha resultado razonablemente barata. Si, como en las revistas americanas del gremio, aportáramos los datos de coste (que, por otra parte, son públicos y conocidos), los primeros asombrados serian algunos de los especialistas eu. ropeos en el tema.

Este aspecto es particularmente reconfortante para quienes negociamos los presupuestos de oferta y para el contribuyente hispano en general, porque, como decia Foxá "... los experimentos, con gaseosa...".

Aunque no he cumplido el compromiso de ser breve, me cabe la excusa del esfuerzo por superar - una vez más y por escrito - mis contradictorios sentimientos respecto de la Torre en cuestión.

Sigo pensando que es en cierto modo injusto - no por lógico menos injusto- que una sola obra asuma la representación de un conjunto de trabajos simultáneos de mayor volumen (económico) y no menor complejidad; que un arquitecto hable en nombre de un equipo entero; que una torre más bien bajita (13) se convierta en simbolo de la propia RTVE (ref.: telediarios, en los que sale torcida y penosamente esquematizada). Pero he de admitir, icebergs aparte, que ha sido una aventura fascinante, un ejercicio imprescindible de colabora. ción interdisciplinar, una muy estimable demostración del nivel de tecnologia nacional $y$, como dicen mis hilos, un "farde".

\section{referencias}

(1) Riesgo arquitectónico y profesional, se entiende.

(2) En el caso de que se esté más o menos de acuerdo con el resultado final.

(3) Se trata de una reserva de superficie que parece razonable a esas alturas y a esos costes de construcción.

(4) Esto es algo de to que estoy particularmente orgulloso, asombrado y agradecido a quienes - con algún esfuerzo extra, supongo- calcularon la estructura propuesta y no otra.

(5) O, mejor, de no mantenimiento.

(6) Algo tuvo que ver el recuerdo de las oxidadas cúpulas de cobre de Copenhague y Estocolmo...

(7) Para tranquilidad de los viandantes y ciudadanos, en general, debo decir que éste no se hubiera autorizado de faltar aquéllas.
(8) Es el caso de la sustitución de la grúa adosada al fuste, por otra situada en la parte superior de éste.

(9) Es un recuerdo tan vivido que no he sido capaz de omitirlo en ninguno de los -.ya bastantes - artículos publicados sobre este asunto de la Torre ly me temo que seguirá apareciendo en los siguientes.

(10) La posibilidad de instalar un elevador mas rapido fue abortada con un planteamiento muy "corto", por parte de RTVE, de las disponibilidades económicas.

(11) Nos gastamos un dinero, pero valio la pena. Ya somos los octavos.

(12) En este sentido, es evidente - a posteriori- la conveniencia de incor porar un equipo de "edificación» a la última fase de construcción. Una torre es, también, un edificio.

(13) Todos - todos - los que en ella hemos intervenido añoramos unos 40/50 metros más... 